A study of the electron structure of endohedrally confined atoms using a model potential

This article has been downloaded from IOPscience. Please scroll down to see the full text article.

2011 J. Phys. B: At. Mol. Opt. Phys. 44015003

(http://iopscience.iop.org/0953-4075/44/1/015003)

View the table of contents for this issue, or go to the journal homepage for more

Download details:

IP Address: 200.128.60.31

The article was downloaded on 05/03/2012 at 19:46

Please note that terms and conditions apply. 


\title{
A study of the electron structure of endohedrally confined atoms using a model potential
}

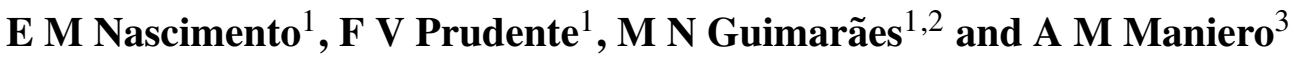 \\ ${ }^{1}$ Instituto de Física, Universidade Federal da Bahia, 40210-340 Salvador, BA, Brazil \\ ${ }^{2}$ Centro de Formação de Professores, Universidade Federal do Recôncavo da Bahia, 45300-000 \\ Amargosa, BA, Brazil \\ ${ }^{3}$ Instituto de Ciências Ambientais e Desenvolvimento Sustentável, Universidade Federal da Bahia, \\ 47805-100 Barreiras, BA, Brazil \\ E-mail: edmar@ufba.br and prudente@ufba.br
}

Received 19 September 2010, in final form 25 October 2010

Published 16 December 2010

Online at stacks.iop.org/JPhysB/44/015003

\begin{abstract}
A novel model potential for modelling the environment of atoms and molecules inside fullerenes is proposed. The model takes into consideration that the electrons of the guest atom or molecule are affected by an attractive short-range Gaussian shell to simulate the $\mathrm{C}_{n}$ cage. As a test case, the present model is employed to study the electronic structure of an endohedrally confined hydrogen atom by $\mathrm{C}_{36}$ and $\mathrm{C}_{60}$ fullerenes. This study is performed using a new implementation of the p-version of the finite-element method by a self-consistent finite-element methodology. The results are then compared with previous ones obtained by using other short-range model potentials.
\end{abstract}

\section{Introduction}

The study of particles, atoms and molecules confined in various environments and their properties has received growing attention in recent years due to the importance of these quantum systems in different physical and chemical fields. For example, these systems are important in mesoscopic-scale semiconductors structures [1,2], where artificial quantum dots with a controlled number of electrons are built [1, 3]; in catalysis when adsorption phenomena are investigated [4]; in the embedding of atoms and molecules inside cavities such as zeolite molecular sieves [5], fullerenes [6, 7], or solvent environments [8]; in bubbles formed around foreign objects in the liquid helium or neutral plasma [9, 10]; in the investigation of atoms under pressure [11-14], for instance. The simplest problem involving atoms and molecules arises from the confinement of the hydrogen atom in a spherical shell [11, 15-23].

In particular, the endohedral fullerenes, systems where atoms and small molecules are confined in a cage of carbon with $\mathrm{C}_{n}(n \geqslant 20)$ [24], have attracted much interest because of their applications in nanoscience and nanotechnology [25]. Various types of these complexes are obtained placing different compounds inside the carbon cage as, for example, noble gases [26-28] and metals [29, 30]. It is interesting to point out that the doping procedure modifies the molecular and solid state properties of the fullerenes. These systems possess great interest due to the recent progress of their synthesis and the multiple technological applications, which go from medicine (see [29] and references therein) to quantum computation [31, 32]. The endohedral fullerenes have been studied experimentally and theoretically by several research groups in more recent years in areas such as physics, chemistry and biology. From the theoretical point of view, to understand the formation process of these complexes and their structures it is necessary to study the electronic structure, the dynamics of guest atoms and molecules and their solid state properties [30].

Usually, the electronic structure studies of endohedral fullerenes are performed considering all electrons by using $a b$ initio density functional theory and semi-empirical methods (see [33, 34] and references therein). However, it can be interesting to substitute 'all electrons' descriptions by models where only electrons of the guest atom or molecule are considered, being affected by an attractive spherical potential that simulates the $\mathrm{C}_{n}$ cage. This strategy has been utilized with 
success to describe the essential features of the experimental results $[35,36]$. The most employed models are the shortrange potential shell of inner radius $r_{c}$ and thickness $\Delta[35$, 37-43],

$$
V_{\mathrm{sr}}(r)= \begin{cases}-U_{0} & \text { for } r_{c} \leqslant r \leqslant r_{c}+\Delta, \\ 0 & \text { otherwise },\end{cases}
$$

and the $\delta$-potential [7, 44-47]

$$
V_{\delta}(r)= \begin{cases}-U_{0} \delta(r) & \text { for } r=r_{c}, \\ 0 & \text { otherwise. }\end{cases}
$$

Potentials (1) and (2) can introduce numerical instability due to their sharp forms, and proposals have been made to avoid it [48, 49].

In this paper, a novel model potential is proposed that has a smooth form to describe the confinement of an atom inside a fullerene cage. This potential consists in an attractive short-range spherical Gaussian shell that is added in the 'free' atomic Hamiltonian. The advantage with such a potential is that it continuously shows a more realistic description of the confinement. As a test case, the Gaussian shell model is utilized to study the endohedrally confined hydrogen $(\mathrm{H})$ atom by a $C_{n}$ cage with $n=36$ and 60 .

The p-version of the finite-element method (p-FEM) $[50,51]$ is used to obtain the solutions (eigenenergies and eigenfunctions) of the associated Schrödinger equation. Recently, the p-FEM with an equidistant mesh was employed to study the hydrogen atom confined by an infinite spherical potential barrier [11]. In particular, we employ the p-FEM with an optimized mesh from a small modification of the quantum mechanical procedure previously proposed [52] that here is denoted by the self-consistent finite-element method (SC-FEM)

This paper is organized as follows. In section 2 we establish the general problem of confined quantum systems and present the spherical Gaussian shell model potential to describe the endohedral atom. In section 3 we give a brief description of the p-FEM and propose the self-consistent procedure to optimize the mesh of elements. Next, we present the main results of this paper in section 4 , comparing them with other previously published results in the literature. Finally, the concluding remarks are given.

\section{The confinement potential}

The general expression of the $N$-electron Hamiltonian for an atomic or molecular confined quantum system, in atomic units, is given by

$$
\hat{H}=\sum_{k}^{N} \hat{h}(k)+\sum_{k<l}^{N} \frac{1}{r_{k l}},
$$

where $\hat{h}(k)$ is the one-electron Hamiltonian which takes into account the kinetic energy, electron-nucleus interaction and the potential of confinement $\hat{w}\left(\vec{r}_{k}\right)$ as follows:

$$
\hat{h}(k)=-\frac{1}{2} \nabla_{k}^{2}-\sum_{A} \frac{Z_{A}}{r_{k A}}+\hat{w}\left(\vec{r}_{k}\right),
$$

and the second part of equation (3) represents the electronelectron repulsion interactions. In the literature, different

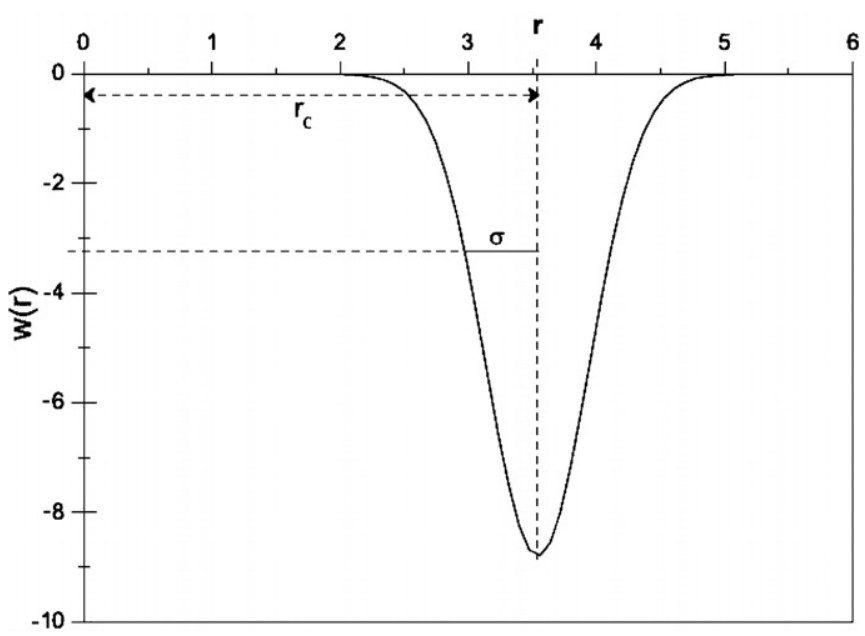

Figure 1. One-dimensional representation of the confinement potential; distance in $\AA$ and potential in $\mathrm{eV}$.

types of potentials have been used to describe the confinement such as harmonic oscillator, spherical and cubic boxes with infinite walls, spherical shells, etc (see, for instance, [3, 36] and references therein). In all of these cases, the main goal is to describe the behaviour and structure of atoms or electrons submitted to the different types of confinement.

In the present approach to simulate the environment of a fullerene cage, the confinement is modelled by an attractive short-range spherical Gaussian-type potential given by

$$
\hat{w}(r)=-w_{0} \exp \left[-\left(r-r_{c}\right)^{2} / \sigma^{2}\right],
$$

where $r=|\vec{r}|, w_{0}$ is the maximal amplitude of the well depth, $r_{c}$ is the radius of the centre of the confinement shell, taken from the origin to the maximum of $\hat{w}$, and $\sigma$ is defined as the half-width at the $w_{0} e^{-1}$ amplitude. The model potential is proposed in order to give a more realistic description of the physical behaviour of the confinement environment than the previous ones (equations (1) and (2)). The Gaussian function and its derivatives are continua, and it is easy to integrate over the whole region of interest. Moreover, fullerenes with a different number of carbons can be modelled by the adjustment of the confinement shell radius, $r_{c}$, the thickness, given by $\sigma$, and the well depth, $w_{0}$. A one-dimensional representation of the potential is given in figure 1 , for $r_{c}=3.54 \AA, w_{0}=8.80$ $\mathrm{eV}$ and $\sigma=0.57 \AA$.

For the specific case of the endohedrally confined hydrogen atom at the centre of the $\mathrm{C}_{n}$ cage, the one-electron problem can be described by the following non-relativistic time-independent Schrödinger equation:

$$
\hat{H} \Psi(\vec{r}) \equiv\left[-\frac{1}{2} \nabla^{2}-\frac{1}{r}+\hat{w}(r)\right] \Psi(\vec{r})=E \Psi(\vec{r}),
$$

where $E$ and $\Psi(\vec{r})$ are respectively the eigenenergy and eigenfunction of the problem. This equation is solved by using a p-FEM, which is described in the following section. 


\section{The finite-element method}

\section{1. $p$-version of FEM}

The FEM is a general nomenclature for a set of different procedures $[53,54]$ which are based on the technique of space discretization into elements and in the use of local polynomial basis functions defined on these elements. In this paper a variational procedure is considered where the p-version of the FEM is employed as the finite basis set in the expansion of the wavefunction (see, for instance, $[11,50-52,55]$ ).

According to the variational principle, the problem to solve equation (6) is equivalent to finding stationary solutions of a functional of energy,

$$
J[\Psi]=\int \Psi^{*}(\vec{r})\{\hat{H}-E\} \Psi(\vec{r}) \mathrm{d} \vec{r},
$$

$\hat{H}$ being the Hamiltonian expressed in equation (6). Since the potential is radial, the wavefunction can be expanded by using the spherical harmonics,

$$
\Psi(\vec{r})=\sum_{l} a^{l} \frac{\psi^{l}(r)}{r} Y_{l m}(\theta, \phi) .
$$

Using the orthonormalization relations of $Y_{l m}(\theta, \phi)$, functional (7) can be written in terms of $\psi_{l}(r)$ as follows:

$$
J[\Psi]=\sum_{l}\left|a_{l}\right|^{2} J_{l}\left[\psi_{l}\right],
$$

where $l$ is the total angular momentum, the functional $J\left[\psi_{l}\right]$ is expressed as

$$
J_{l}\left[\psi_{l}\right]=\int_{0}^{r_{\max }} \mathrm{d} r\left\{\frac{1}{2} \frac{\mathrm{d} \psi_{l}^{*}}{\mathrm{~d} r} \frac{\mathrm{d} \psi_{l}}{\mathrm{~d} r}+\psi_{l}^{*}\left[V_{l}^{\mathrm{ef}}(r)-E\right] \psi_{l}\right\}
$$

and $V_{l}^{\text {ef }}(r)=-1 / r+\hat{w}(r)+l(l+1) / 2 r^{2}$. Dirichlet's boundary conditions were utilized to obtain equation (10). The eigenstate and eigenvalue solutions of $J_{l}\left[\psi_{l}\right]$ are found by expanding the wavefunction in a finite basis set $\left\{f_{j}^{l}\right\}$, and imposing the stationarity condition on functional (10) under consideration. Thus, the variational solutions are obtained by solving the resultant generalized eigenvalue-eigenvector problem.

Computational effort to solve the generalized eigenvalueeigenvector problem depends directly on the number of basis functions used to expand $\psi_{l}(r)$. Therefore, the efficiency of numerical calculation depends on the choice of the finite basis set. In particular, the one-dimensional p-FEM is employed for this purpose. The procedure consists of dividing the integration interval $\left[0, r_{\max }\right]$ into $N_{e}$ elements, where the $i$ th element has a range of $r_{i-1}$ to $r_{i}$ with $r_{0}=0$ and $r_{N_{e}}=r_{\max }$, defining the local basis functions $\left\{f_{j}^{i}\right\}$, which are non-null only on the $i$ th element, and expanding the wavefunction as follows:

$$
\psi_{l}(r)=\sum_{i=1}^{N_{e}} \sum_{j=0}^{k_{i}} c_{j}^{l, i} f_{j}^{i}(r) .
$$

The p-FEM utilizes, as local basis functions, two interpolants $\left(f_{0}^{i}(r)\right.$ and $\left.f_{k_{i}}^{i}(r)\right)$ and $k_{i}-1$ shape functions $\left(f_{j}^{i}(r), j=1, \ldots, k_{i}-1\right)$ that have the following properties:

$$
\begin{aligned}
& f_{0}^{i}\left(r_{i-1}\right)=1=f_{k_{i}}^{i}\left(r_{i}\right) \\
& f_{0}^{i}\left(r_{i}\right)=0=f_{k_{i}}^{i}\left(r_{i-1}\right)
\end{aligned}
$$

$$
f_{j}^{i}\left(r_{i-1}\right)=0=f_{j}^{i}\left(r_{i}\right), \quad j=1, \ldots, k_{i}-1
$$
and

$$
\int_{0}^{r_{\max }} \mathrm{d} r f_{j}^{i *}(r) f_{j^{\prime}}^{i^{\prime}}(r)=0, \quad \forall i \neq i^{\prime} .
$$

Moreover, the imposition of the continuity of the wavefunction on the border of elements leads to the condition $c_{k_{i}}^{i}=c_{0}^{i+1}$ and $f_{k_{i}}^{i}(r)+f_{0}^{i+1}(r)$ being only one function actuating in two elements. Thus, a new independent coefficient set $\left\{a_{k}\right\}$ is defined from $\left\{c_{j}^{l, i}\right\}$ as follows:

$$
a_{k}^{l}=c_{j}^{l, i} \quad \Longleftrightarrow \quad k=(i-1) k_{i}+j,
$$

totalizing $N=\sum_{i=1}^{N_{e}} k_{i}$ basis functions; $c_{0}^{l, 1}=a_{0}^{l}=0$ due to the continuity of the wavefunction (equation (8)) at the origin. Using these relations, the matrix representation $\mathbf{B}$ of any local operator $\mathcal{B}$ has a useful block structure, is very sparse and, if $\mathcal{B}$ is Hermitian, is real and symmetric.

\subsection{Self-consistent FEM}

One important aspect of the FEM is the choice of $\left\{r_{i}\right\}, i=0, \ldots, N_{e}$, points that define the elements of the unidimensional mesh. The simplest mesh is the one obtained from an equidistant discretization as follows:

$$
r_{i}=i \frac{r_{\max }}{N_{e}}
$$

However, in many situations using an optimized mesh in order to obtain accurate results with a lesser number of basis functions can be interesting. Previously, Prudente and Soares Neto introduced a quantum mechanical procedure (QMP) to build the optimized mesh for an arbitrary potential [52]. The QMP was motivated by the potential-optimized discrete variable representation (PO-DVR) method [56-59]. In such a method, the Gaussian quadrature points are the eigenvalues of the position operator in a specific orthonormal basis function.

Here a modification on the QMP is proposed transforming it in a self-consistent procedure to solve one-dimensional quantum problems employing the p-version of the FEM. The basic steps of the self-consistent FEM are given as follows.

(i) Solve the one-dimensional problem using the p-FEM with a (small) equidistant mesh and determine an approximate set of eigenvalues $\left\{E_{i}\right\}$ and eigenfunctions $\left\{\phi_{i}(r)\right\}$.

(ii) Using the $N_{e}-1$ first eigenfunctions, build the $\mathbf{X}$ matrix whose elements are given by

$$
\{\mathbf{X}\}_{i j}=\int_{0}^{r_{\max }} \mathrm{d} r \phi_{i}^{*}(r) r \phi_{j}(r),
$$

where $\mathbf{X}$ is the matrix representation of the position operator.

(iii) Diagonalize $\mathbf{X}$. The $N_{e}-1$ eigenvalues are the $r_{1}, r_{2}, \ldots, r_{N_{e}-1}$ points that define the borders of elements ( $r_{0}=0$ and $r_{N_{e}}=r_{\max }$ are previously chosen).

(iv) Solve the one-dimensional problem using the p-FEM with the mesh determined in step (iii) to obtain the new eigenvalues and eigenfunctions. If the absolute difference between the old and new eigenvalues is greater than a desirable tolerance, go to step (ii) and repeat the procedure. When the convergence is reached, the iterative process is concluded and the final optimized mesh is obtained. 
Table 1. Eigenvalues with energy levels of $1 \mathrm{~s}$ to $4 \mathrm{~s}$ states for different values of $w_{0}$ and $\sigma$ using the SC-FEM.

\begin{tabular}{|c|c|c|c|c|c|}
\hline \multirow[b]{2}{*}{$w_{0}(\mathrm{Ryd})$} & \multirow[b]{2}{*}{$\sigma(\AA)$} & \multicolumn{4}{|c|}{ Energy levels (hartree) } \\
\hline & & $1 \mathrm{~s}$ & $2 \mathrm{~s}$ & $3 \mathrm{~s}$ & $4 \mathrm{~s}$ \\
\hline 1.000 & 0.26 & -0.5002 & -0.2224 & -0.0565 & -0.0316 \\
\hline 1.000 & 0.57 & -0.5013 & -0.3418 & -0.0639 & -0.0362 \\
\hline 1.000 & 1.59 & -0.5584 & -0.4892 & -0.2480 & -0.0708 \\
\hline 0.647 & 0.26 & -0.5001 & -0.1804 & -0.0562 & -0.0314 \\
\hline 0.647 & 0.57 & -0.5006 & -0.2504 & -0.0599 & -0.0336 \\
\hline 0.647 & 1.59 & -0.5280 & -0.3603 & -0.1594 & -0.0491 \\
\hline 0.324 & 0.26 & -0.5000 & -0.1495 & -0.0559 & -0.0313 \\
\hline 0.324 & 0.57 & -0.5002 & -0.1799 & -0.0575 & -0.0322 \\
\hline 0.324 & 1.59 & -0.5128 & -0.2382 & -0.0918 & -0.0406 \\
\hline \multicolumn{2}{|c|}{ ee } & -0.5000 & -0.1250 & -0.0556 & -0.0312 \\
\hline
\end{tabular}

\section{Results}

In this section the SC-FEM is employed to perform the electronic structure calculations of a hydrogen atom inside $\mathrm{C}_{60}$ and $\mathrm{C}_{36}$ fullerenes modelled by the Gaussian shell potential (5). All SC-FEM calculations have been performed using $N_{e}=20, k_{i}=8 \forall i$, totalizing 139 local basis functions, and $r_{\max }=200.0 a_{0}$; this asserts an accuracy of, at least, four figures in the energy levels. On the other hand, the model potential has three adjustable parameters $\left(w_{0}\right.$, maximum well depth; $r_{c}$, centre of confinement shell; $\sigma$, half-width) that must be chosen appropriately to simulate the cages. An obvious choice of $r_{c}$ is taken equal to the characteristic (or experimental) radius of the fullerene cage; here $r_{c}=3.54 \AA$ is utilized for $\mathrm{C}_{60}$ [35] and $r_{c}=2.50 \AA$ for $\mathrm{C}_{36}$ [60]. The other two parameters should then be obtained from other experimental and theoretical information about the fullerenes.

In order to show the adaptation of the present model potential, the energy values for the first four states for $l=0$ of the confined hydrogen atom are calculated for different values of $w_{0}$ and $\sigma$, assuming $r_{c}$ in its $\mathrm{C}_{60}$ value. These results are shown in table 1 , jointly with the respective energies of the free hydrogen atom calculated with the SC-FEM. We can note that each energy level has a different behaviour when the confinement parameters are changed. The electronic state that suffers the highest influence is the $2 \mathrm{~s}$ one, while the states $1 \mathrm{~s}$ and $4 \mathrm{~s}$ are almost not modified within the range of parameters shown in table 1 . These results indicate the flexibility of the Gaussian model potential.

Moreover, it is interesting to point out the importance of the optimization process of the element mesh at SC-FEM. In this procedure, initially an equidistant mesh is proposed and, after some self-consistent cycles, a new FEM mesh optimized for the potential is obtained. For example, figure 2 displays the equidistant and the optimized mesh, jointly with the effective (Coulombic + Gaussian confinement + centrifugal) potential, $V_{l}^{\text {ef }}(r)$, for $w_{0}=0.647 \mathrm{Ryd}, \sigma=0.57 \AA, r_{c}=3.54 \AA$ and $l=0$. As expected, in the figure it can be clearly seen that the node points of the optimized mesh are more concentrated in the regions where the potential reaches a minimum. The improvement in the energy values is great: for the $1 \mathrm{~s}$ energy level, the equidistant mesh gives a value of -0.4987 hartree,

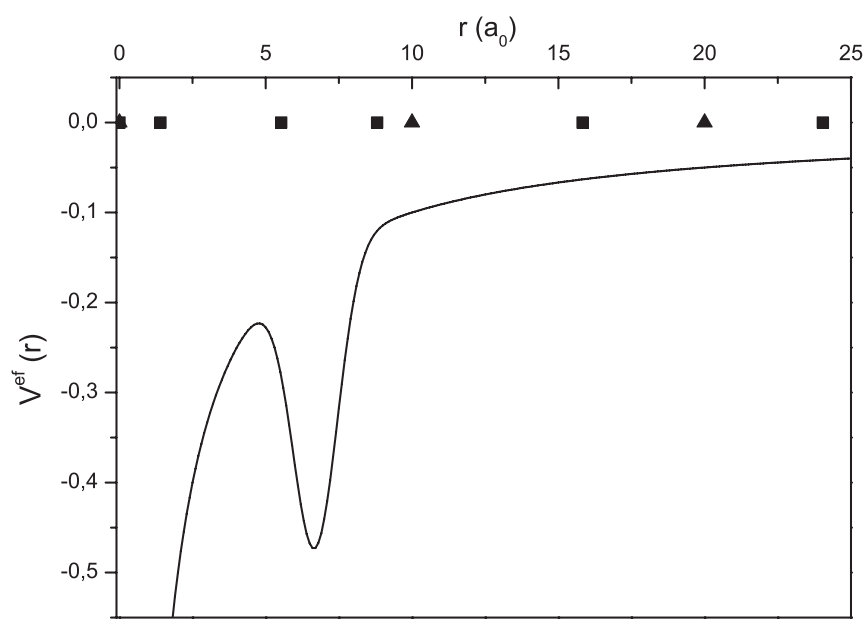

Figure 2. The effective (Coulombic + Gaussian confinement) potential $V_{0}^{\text {ef }}(r)$ (full line), the equidistant (triangle up) mesh and the optimized one (square) obtained using the SC-FEM; distance in bohr and potential energy in hartree.

while for the optimized one, the accurate value is found to be -0.5006 hartree.

The next step is to compare the Gaussian shell model with the previous one, equation (1), proposed by Connerade et al [38]. For this purpose, the energy levels of $n \mathrm{~s}, n=1, \ldots, 4$, electronic states for $\mathrm{H} @ \mathrm{C}_{60}$ are calculated as a function of the confining well depth, $w_{0}$. In order to compare both models, the value of $\sigma$ in the present model is adjusted to satisfy the condition

$$
\int_{0}^{\infty} \hat{w}(r) \mathrm{d} r=\int_{0}^{\infty} V_{\text {sr }}(r) \mathrm{d} r=-U_{0} \Delta
$$

where $U_{0}$ is the well depth of the square well and $\Delta$ is the thickness of equation (1), while it is assumed that $w_{0}=U_{0}$. Following Connerade et al [38], it is assumed that $\Delta=1 \AA$, leading $\sigma=0.57 \AA$ to the $\mathrm{C}_{60}$ cage. This choice is rather arbitrary, and there are other possibilities. One eventually could more naturally utilize the volume element in spherical coordinates, $r^{2} \mathrm{~d} r$. However, in such a case the value of $\sigma$ practically does not change, leading to the similar results obtained by using equation (18).

The results of the spherical Gaussian shell are shown in figure 3, where they are compared with those obtained by Connerade et al [38]. We can see good agreement between both results, and the observed differences are due to the functional differences among the confinement potentials. For example, the 2 s state is strongly modified even for small values of $w_{0}$, while the other states remain practically unchanged. Moreover, the avoided crossings between the $n \mathrm{~s}$ and $(n+1) \mathrm{s}$ states remain in the novel model, but their localizations are slightly different; the $1 \mathrm{~s}-2 \mathrm{~s}, 2 \mathrm{~s}-3 \mathrm{~s}$ and $3 \mathrm{~s}-4 \mathrm{~s}$ avoided crossings occur approximately at 1.5 Ryd, 5.4 Ryd and 11.6 Ryd, respectively, for the present potential, while for the Connerade et al potential these crossings occur approximately at $1.4 \mathrm{Ryd}$, 5.0 Ryd and 13.5 Ryd, respectively.

To analyse the effect of the confinement cage in states with $l \neq 0$, figure 4 displays the 1 s to $4 \mathrm{~d}$ energy levels of $\mathrm{H} @ \mathrm{C}_{60}$ as a function of $w_{0}$ calculated using the Gaussian shell. The 


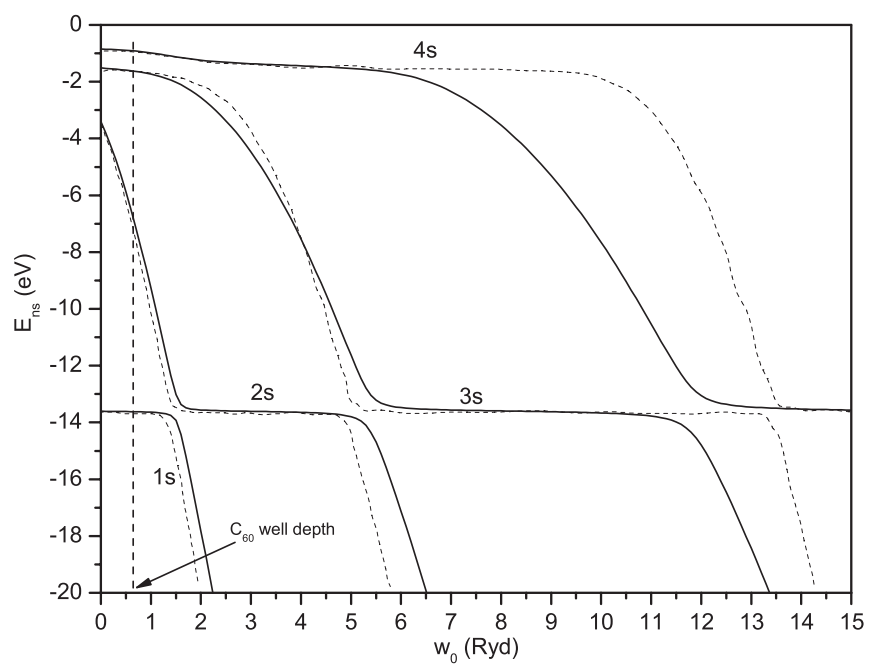

Figure 3. Energies of $1 \mathrm{~s}$ to $4 \mathrm{~s}$ levels of $\mathrm{H} @ \mathrm{C}_{60}$ as a function of $w_{0}$. Solid lines, our results; dashed lines, Connerade et al [38] results. The vertical dashed line indicates the value of $w_{0}$ adjusted by the electron affinity of $\mathrm{C}_{60}$.

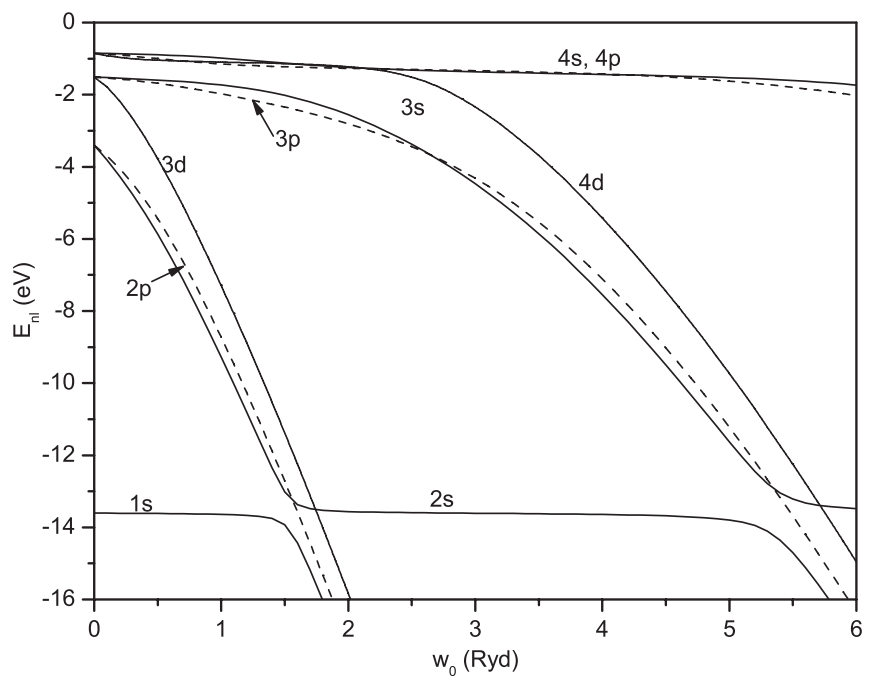

Figure 4. The $1 \mathrm{~s}$ to $4 \mathrm{~d}$ energy levels of $\mathrm{H} @ \mathrm{C}_{60}$ as a function of $w_{0}$ calculated using the SC-FEM.

splitting of the degeneracy observed in the free hydrogen atom for the levels $n l$, with $0 \leqslant l \leqslant n-1$, due to the confinement potential can be noted clearly. The $n l$ states more affected by the fullerene cage are the ones with $l=n-1$. Thus, the level ordering is seen to be $1 \mathrm{~s}, 2 \mathrm{p}, 3 \mathrm{~d}, 2 \mathrm{~s}, 3 \mathrm{p}$ and $3 \mathrm{~s}$ states at $w_{0}=2.0$ Ryd.

As commented previously, the potential depth $w_{0}$ to simulate a real $\mathrm{C}_{60}$ cage can be obtained from experimental or theoretical data. A simple procedure is to fit the theoretical valuation of the electron affinity to the experimental one [36]. The theoretical electron affinity can be calculated by solving the one-electron problem placed inside the Gaussian attractive shell (equation (6)) without the $-1 / r$ term. For an electron affinity of $2.65 \mathrm{eV}$ for $\mathrm{C}_{60}$ [61], we have obtained $w_{0}=8.80 \mathrm{eV}=0.647 \mathrm{Ryd}$ for the present model, while for the Connerade et al model potential, the well depth is found to be $U_{0}=8.22 \mathrm{eV}$

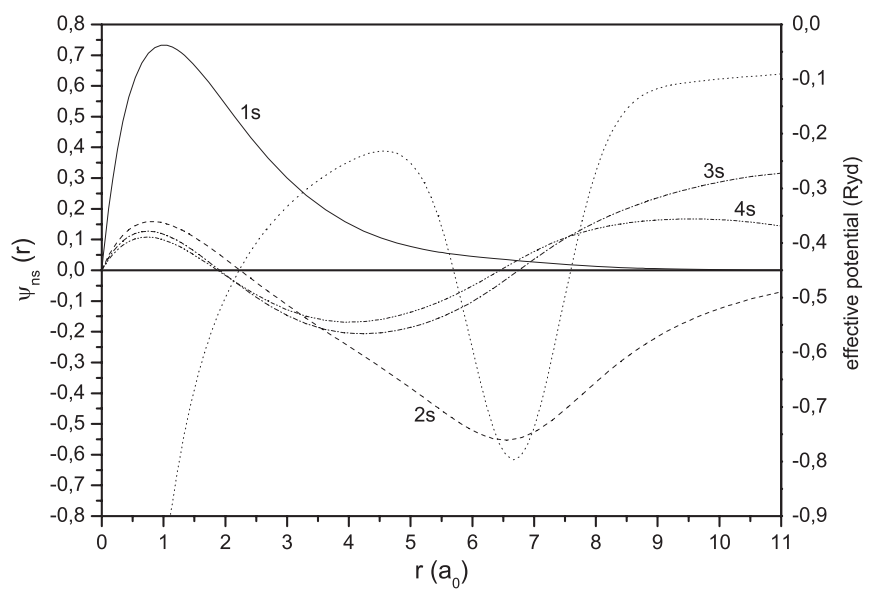

Figure 5. The radial $\psi_{n s}$ wavefunctions of $\mathrm{H} @ \mathrm{C}_{60}$ at $w_{0}=$ 0.647 Ryd and the effective (Coulombic + Gaussian confinement) potential. Solid lines, 1s state; dashed line, 2s state; dashed dotted line, $3 \mathrm{~s}$ state; dashed dotted dotted line, $4 \mathrm{~s}$ state; dotted line, effective (Coulombic + Gaussian confinement) potential.

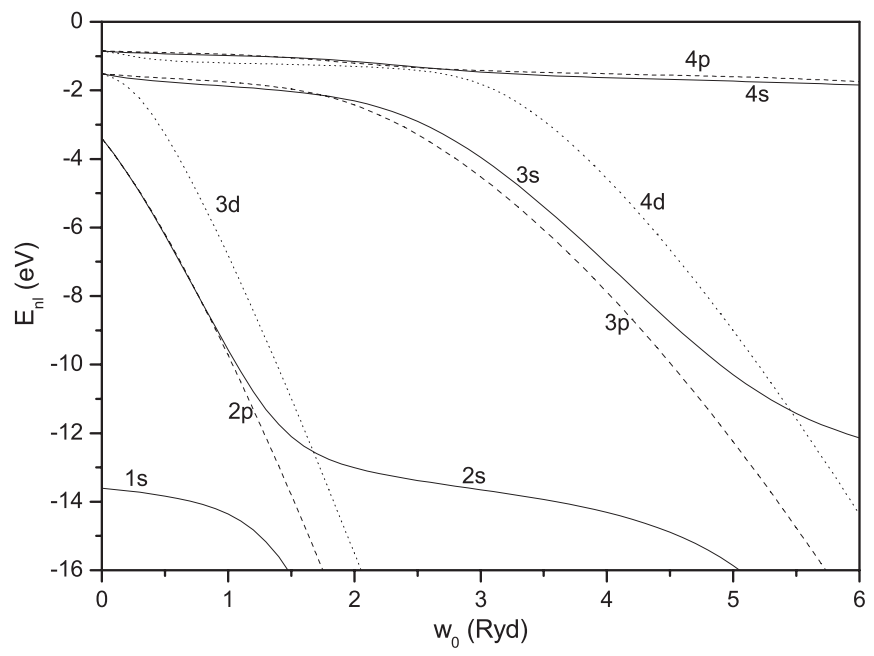

Figure 6. The $E_{1 \mathrm{~s}}$ to $E_{4 \mathrm{~d}}$ energy levels of $\mathrm{H} @ \mathrm{C}_{36}$ as a function of $w_{0}$ calculated using the SC-FEM.

[36]. In particular, the wavefunction of the $n$ s states, $n=1, \ldots, 4$, and the effective (Gaussian confinement + Coulombic) potential with $l=0$ calculated at a value of $w_{0}=0.647 \mathrm{Ryd}(8.80 \mathrm{eV})$ are displayed in figure 5. Thus, we can see that the states $1 \mathrm{~s}, 3 \mathrm{~s}$ and $4 \mathrm{~s}$ remain bound in the inner Coulomb shell, while the $2 \mathrm{~s}$ state has great amplitude in the confinement region. We can easily recognize that the $2 \mathrm{~s}$ state is strongly bounded by the confinement, while the others remain bounded in the inner Coulomb well.

In order to show the versatility of the attractive Gaussian shell model potential, we also apply it in the study of the electronic structure of $\mathrm{H} @ \mathrm{C}_{36}$. So we show, in figure 6, the 1s to $4 \mathrm{~d}$ energy levels of $\mathrm{H} @ \mathrm{C}_{36}$ as a function of $w_{0}$. The avoided crossings between the $n \mathrm{~s}$ and $(n+1) \mathrm{s}$ states are still perceived but less evident than in the $\mathrm{H} @ \mathrm{C}_{60}$ case. This indicates that the coupling between Coulombic and confinement cage potentials is more intense in the present system. The stronger confinement over the states of $\mathrm{H} @ \mathrm{C}_{36}$ is clearly related to the 


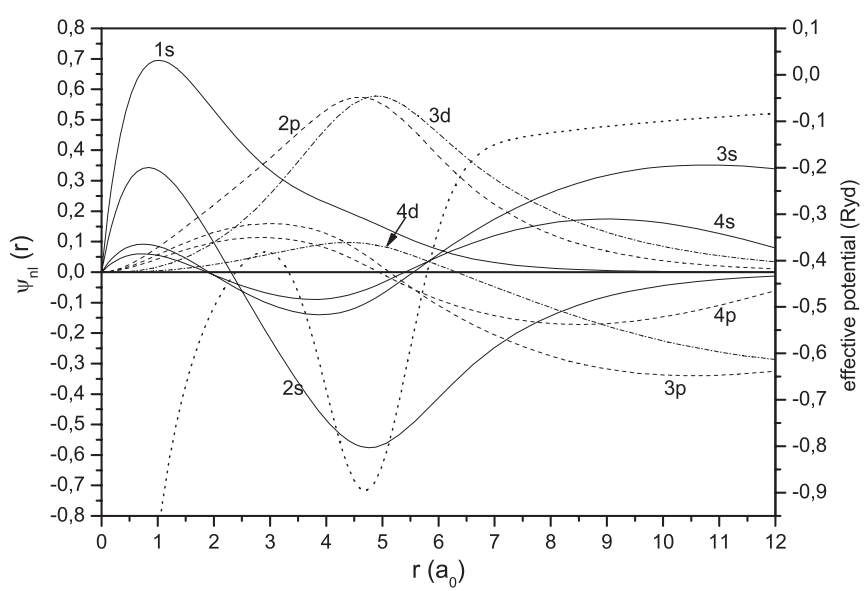

Figure 7. The radial wavefunctions of $1 \mathrm{~s}$ to $4 \mathrm{~d}$ states for $\mathrm{H} @ \mathrm{C}_{36}$ at $w_{0}=0.683$ Ryd and the effective potential. Solid lines, s states; dashed lines, $\mathrm{p}$ states; dashed dot lines, $\mathrm{d}$ states; dotted line, effective (Coulombic + Gaussian confinement) potential.

smallest radius of the $\mathrm{C}_{36}$ molecule. Moreover, the splitting of the degeneracy for the levels $n l$, with $0 \leqslant l \leqslant n-1$, due to the confinement potential is also observed.

By evaluating the potential depth $w_{0}$ to simulate a real $\mathrm{C}_{36}$ cage, we have found $w_{0}=9.29 \mathrm{eV}=0.683 \mathrm{Ryd}$ by considering the value of $2.8 \mathrm{eV}$ for the experimental electron affinity of $\mathrm{C}_{36}$ [62]. We can see that, at a typical value of $w_{0}=0.683$ Ryd for $\mathrm{C}_{36}$, all states feel the influence of the confinement well. To show this more clearly, figure 7 displays the $1 \mathrm{~s}$ to $4 \mathrm{~d}$ wavefunctions calculated at this $w_{0}$, and the effective (Coulombic + Gaussian confinement) potential $V_{0}^{\text {ef }}(r)$. The states $2 \mathrm{~s}, 2 \mathrm{p}, 3 \mathrm{~d}$ have most of their amplitudes in the confinement region and remain bound in the outer well. The $3 \mathrm{~s}, 4 \mathrm{~s}, 3 \mathrm{p}, 4 \mathrm{p}$ and $4 \mathrm{~d}$ states appear partially bound in the outer confining well. On the other hand, the declines of the curves in figure 6 show that for greater values of $w_{0}$, the $2 \mathrm{~s}, 3 \mathrm{~s}$ and $4 \mathrm{~s}$ levels do not become completely confined in the inner Coulomb well as we saw for $\mathrm{C}_{60}$. The $1 \mathrm{~s}$ state has most of its amplitude in the inner Coulomb well but a significant amount of it in the outer well also, so that the energy is decreased by an amount nearly equal to the confining well depth.

\section{Conclusion}

In this paper, a new model potential is proposed in order to describe the confinement of atoms inside fullerene cages. This procedure was applied to the confinement of the hydrogen atom inside $\mathrm{C}_{60}$ and $\mathrm{C}_{36}$ molecules, and the results have been compared to the others found in the literature. Particularly, we choose the results obtained by Connerade et al [38] in a very similar situation with ours to perform a detailed comparison. Our calculations have shown that, to the typical well depth for $\mathrm{C}_{60}$, most of the $n$ s states stay bound in the inner Coulomb shell, while for $\mathrm{C}_{36}$ all the states feel the influence of the confinement well; this is related to the smallest radius of the $\mathrm{C}_{36}$ cage. We believe that this attractive short-range spherical Gaussian potential type resembles better the physical confinement than a more simple short-range spherical potential. Moreover, the use of Gaussian functions is convenient in calculations involving the integration of the potential. So this model potential can be easily applied to other nearly spherical systems and multielectronic atoms. In particular, studies of the photoionization process of the endohedrally confined atom by the fullerene cage are being carried through and the results will be published in the near future.

\section{Acknowledgments}

This work has been supported by the following Brazilian National Research Councils: Conselho Nacional de Desenvolvimento Científico e Tecnológico (CNPq), Coordenação de Aperfeiçoamento de Pessoal de Nível Superior (CAPES) and Fundação de Amparo a Pesquisa do Estado da Bahia (FAPESB).

\section{References}

[1] Jacak L, Hawrylak O and Wojs A 1998 Quantum Dots (Berlin: Springer)

[2] Zrenner A 2000 J. Chem. Phys. 1127790

[3] Prudente F V, Costa L S and Vianna J D M 2005 J. Chem. Phys. 123224701

[4] Frank K H, Didde R, Sagner H J and Eberhardt W 1989 Phys. Rev. B 39940

[5] Tang Z K, Nozue Y and Goto T J 1992 J. Phys. Soc. Japan 612943

[6] Cioslowski J and Fleischmann E D 1991 J. Chem. Phys. 943730

[7] Baltenkov A S 1999 J. Phys. B: At. Mol. Opt. Phys. 322745

[8] Reichardt C 1988 Solvents and Solvents Effects in Organic Chemistry (Weinheim: Wiley-VCH)

[9] Saha B, Mukherjee T K, Mukherjee P K and Diercksen G H F 2002 Theor. Chem. Acc. 108305

[10] Mukherjee P K, Karwowski J and Diercksen G H F 2002 Chem. Phys. Lett. 363323

[11] Guimarães M N and Prudente F V 2005 J. Phys. B: At. Mol. Opt. Phys. 382811

[12] Banerjee A, Kamal C and Chowdhury A 2006 Phys. Lett. A 350121

[13] Flores-Riveros A and Rodriguez-Contreras A 2008 Phys. Lett. A 3726175

[14] Wilson C L, Montgomery Jr H E, Sen K D and Thompson D C 2010 Phys. Lett. A 3744415

[15] Michels A, de Boer J and Bijl A 1937 Physica 4981

[16] Sommerfeld A and Welker H 1938 Ann. Phys. (Leipzig) 424 56-65

[17] Goldman S and Joslin C 1992 J. Phys. Chem. 966021

[18] Varshni Y P 1997 J. Phys. B: At. Mol. Opt. Phys. 30 L589

[19] Costa L S, Prudente F V, Acioli P H, Soares Neto J J and Vianna J D M 1999 J. Phys. B: At. Mol. Opt. Phys. 322461

[20] Rivelino R and Vianna J D M 2001 J. Phys. B: At. Mol. Opt. Phys. 34 L645

[21] Banerjee A, Sen K D and Vargas R 2002 J. Chem. Phys. 1164054

[22] Sen K D 2005 J. Chem. Phys. 122194324

[23] Sen K D, Pupyshev V I and Montgomery Jr H E 2009 Adv. Quantum Chem. 5725

[24] Akasaka T and Nagase S 2003 Endofullerenes: A New Family of Carbon Clusters (Dordrecht: Kluwer)

[25] Dillon A C, Jones K M, Bekkedahl T A, Kiang C H, Bethune D S and Heben M J 1997 Nature 386377 
[26] Saunders M, Jiménez-Vázquez H A, Cross R J and Poreda R J 1993 Science 2591428

[27] Patchkovskii S and Thiel W 1996 J. Am. Chem. Soc. 1187164

[28] Darzynkiewicz R B and Scuseria G E 1997 J. Phys. Chem. A 1017141

[29] Shinohara H 2000 Rep. Prog. Phys. 63843

[30] Bethune D S, Johnson R D, Salem J R, de Vries M S and Yannoni C S 1993 Nature 366123

[31] Delaney P and Greer J C 2004 Appl. Phys. Lett. 84431

[32] Connerade J P and Solov'yov A V 2005 J. Phys. B: At. Mol. Opt. Phys. 38807

[33] Cioslowski J 1995 Electronic Structure Calculations on Fullerenes and Their Derivatives (New York: Oxford University Press)

[34] Guha S and Nakamoto K 2005 Coord. Chem. Rev. 2491111

[35] Xu Y B, Tan M Q and Becker U 1996 Phys. Rev. Lett. 763538

[36] Dolmatov V K, Baltenkov A S, Connerade J P and Manson S T 2004 Radiat. Phys. Chem. 70417

[37] Pushka M J and Niemenen R M 1993 Phys. Rev. B 471181

[38] Connerade J P, Dolmatov V K, Lakshmi P A and Manson S T 1999 J. Phys. B: At. Mol. Opt. Phys. 32 L239

[39] Mitnik D M, Randazzo J and Gasaneo G 2008 Phys. Rev. A 78062501

[40] Ndengué S A and Motapon O 2008 J. Phys. B: At. Mol. Opt. Phys. 41045001

[41] Dolmatov V K, Brewer P and Manson S T 2008 Phys. Rev. A 78013415

[42] Dolmatov V K, Craven G T, Guler E and Keating D 2009 Phys. Rev. A 80035401

[43] Ludlow J A, Lee T G and Pindzola M S 2010 Phys. Rev. A 81023407
[44] Amusia M Y, Baltenkov A S and Becker U 1998 Phys. Lett. A 24399

[45] Bekkerman A, Tsipinyuk B and Kolodney E 2002 J. Chem. Phys. 11610447

[46] Patil S H 2005 J. Chem. Phys. 122064302

[47] Amusia M Y, Chernysheva L V and Liverts E Z 2009 Phys. Lett. A 80032503

[48] Lyras A and Bachau H 2005 J. Phys. B: At. Mol. Opt. Phys. 381119

[49] Madjet M E, Chakraborty H S, Rost J M and Manson S T 2008 J. Phys. B: At. Mol. Opt. Phys. 41105101

[50] Soares Neto J J and Prudente F V 1994 Theor. Chim. Acta 89415

[51] Prudente F V and Soares Neto J J 1999 Chem. Phys. Lett. 309471

[52] Prudente F V and Soares Neto J J 1999 Chem. Phys. Lett. 30243

[53] Ciarlet P G 1978 The Finite Element Method for Elliptic Problem (Amsterdam: North-Holland)

[54] Ram-Moham L R 2002 Finite Element and Boundary Element Applications in Quantum Mechanics (New York: Oxford University Press)

[55] Guimarães M N and Prudente F V 2010 in preparation

[56] Echave J and Clary D C 1992 Chem. Phys. Lett. 190225

[57] Wei H and Carrington T J 1992 J. Chem. Phys. 973029

[58] Soares Neto J J and Costa L S 1998 Braz. J. Phys. 281

[59] Bitencourt A C P, Prudente F V and Vianna J D M 2007 J. Phys. B: At. Mol. Opt. Phys. 402075

[60] Piskoti C, Yarger J and Zettl A 1998 Nature 393771

[61] Wang L S, Conceicao J, Jim C and Smalley R E 1991 Chem. Phys. Lett. 1825

[62] Yuan L, Yang J, Deng K and Zhu Q 2000 J. Phys. Chem. A 1046666 